\title{
Proposal of Virtual Network Configuration Acquisition Function for Data Center Operations and Management System
}

\author{
Hideki Okita, Masahiro Yoshizawa, Keitaro Uehara, \\ Kazuhiko Mizuno, Toshiaki Tarui, and Ken Naono \\ Central Research Laboratory, Hitachi, Ltd. \\ \{hideki.okita.pf,masahiro.yoshizawa.bt,keitaro.uehara.tb, \\ kazuhiko.mizuno.pq, toshiaki.tarui.my, ken.naono.aw\}@hitachi.com
}

\begin{abstract}
Virtualization technologies have been widely deployed in data centers to improve the system utilization. However, they cause increased workload for operators to clarify the structure of virtual networks in data centers. To reduce the operation time, this paper provides the virtual-network management system which automates the integration of the configurations of the virtual networks. The proposed system collects the configurations from server virtualization platforms and VLAN-supported switches, and integrates these configurations according to the newly developed XML-based management information model for virtual-network configurations. The preliminary evaluations show that the proposed system helps to reduce the time to collect and update the configurations by about 40 percent. This result implies that the proposed system is effective for improving the configuration management process for virtual networks in data centers.
\end{abstract}

\section{Introduction}

In 2013, the scale of the data-center service market in Japan, which was 88 billion dollars in 2009, is expected to reach 140 billion dollars 9. Reducing management costs is the main motivation for the server consolidation in data centers [11. To achieve this server consolidation, servers are being increasingly virtualized. In addition, High-Performance Computing (HPC) platforms are also being virtualized [7].

To operate efficiently data centers in which server-virtualization technology is used, data-center operators have to manage virtual networks as well as virtual machines (VMs). For such virtual-network management, prior works provide several methods for clarifying the structure of virtual networks in accordance with the configurations of virtual LAN (VLAN) 3] switches and network-connection information [8, 10].

However, to reduce the implementation costs of management functions, the servers with server-virtualization functions (virtualized servers) have configurations with their own forms that differ from those of VLAN switches. As a 
result, to manage the virtual-network structure in data centers in which servervirtualization technology is used, operators need to collect data on multiple forms of configurations. Configuration management time thereby increases.

To improve interoperability of management information, DMTF (Distributed Management Task Force) is developing a standard management model [4]. However, this model covers only virtual VLAN switches created on virtualized servers and does not cover physical VLAN switches. Therefore, the configurations of virtualized servers still differ from those of physical network devices.

In the present study, to reduce the time taken to collect the configuration data about virtual networks in a data center, a "virtual-network-configuration acquisition function" has been developed.

\section{Issues in Virtual-Network Configuration Management}

\subsection{Prior Virtual-Network Configuration Management}

In a network without VLAN, which is standardized as IEEE802.1Q, all servers can communicate with each other. On the other hand, in a VLAN-enabled network, multiple and isolated virtual networks can exist on a physical network.

The logical structure of the VLAN-enabled networks varies according to VLAN configurations. Therefore, to clarify the structure of the networks, operators have to manage the VLAN configurations.

Management Information Base (MIB), a part of the Internet Standard Management Framework [5], is a standard approach for defining management information. Fig 1 shows the management procedure for the previously described VLAN configurations through a MIB.

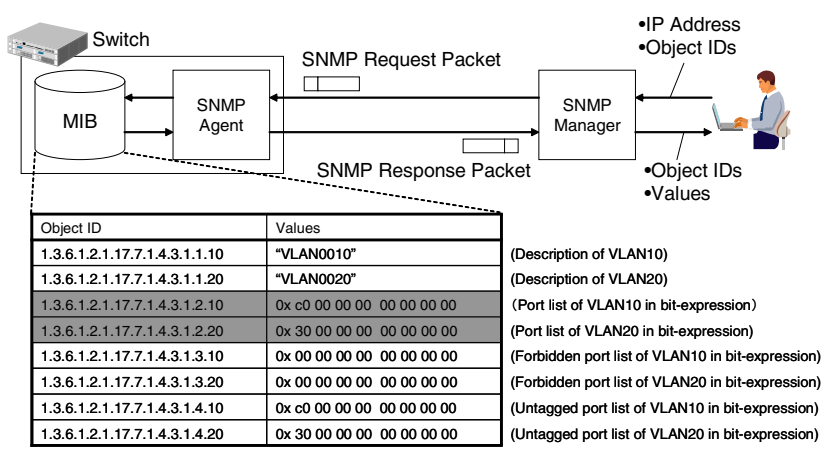

SNMP : Simple Network Management Protocol

Fig. 1. Collecting VLAN configuration with MIB

The two key issues regarding configuration management of virtual networks with MIBs are described as follows.

The first issue concerns the reusability of configuration information. Although the data of MIBs include sets of object IDs and values, MIBs lack information 
about data structure. Therefore, when other management tools reuse VLAN configurations collected through MIBs, they require additional information about the data structure. As a result, the complexity of configuration information causes a decrease in manageability.

The second issue concerns the integrity of configuration information. A MIB contains information only about the switch that the configuration is acquired from. Therefore, as the scale of the managed network expands, the number of VLAN configurations that the operators have to manage increases. In turn, the increased workload to manage the configurations becomes another serious issue.

\subsection{Virtual-Network Configuration Management in Server Virtualization Environments}

Virtualized servers, as shown in Fig,2, run virtual switches to connect VMs on the same server in data centers in which server virtualization is used. Since most of these virtual switches support VLAN technology, the structure of a virtual network varies according to their VLAN configurations.

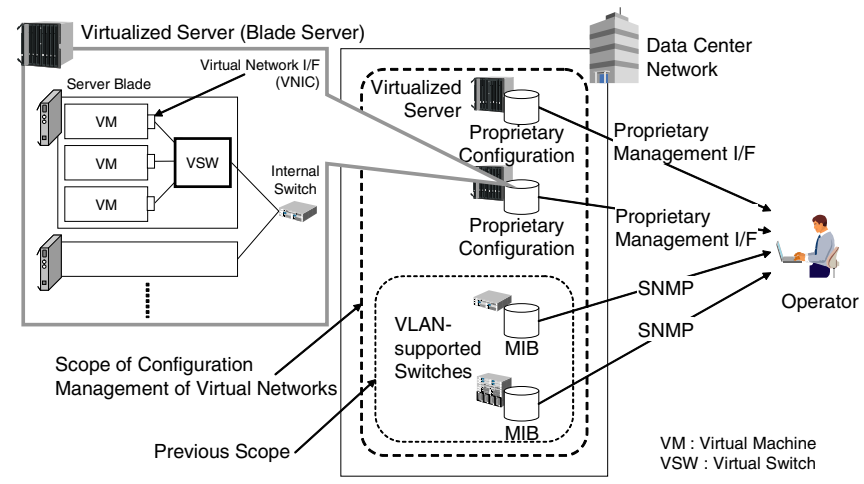

Fig. 2. Virtual network management in the server-virtualized environment

To clarify the structure of virtual networks, the data-center operators therefore have to manage the configurations of the virtualized servers in addition to those of the virtual switches. However, the configurations of the virtualized servers are vendor-specific, unlike those of the Internet-standard MIBs. This makes significant differences in data structure between them. Operators therefore have to manage multiple forms of configurations and thus face increased workload. Additionally, the management interface $(\mathrm{I} / \mathrm{F})$ of the virtualized servers is also a vendor-specific I/F and thus leads to increased workload.

\subsection{Challenges Facing Virtual-Network Configuration Management}

To resolve the three above-described issues, the following three challenges must be faced. First, we should improve the reusability of configuration data so that 
operators can easily recognize its data structure. Second, we should improve the integrity of configurations so that operators can manage multiple devices as a whole. Third, we should improve the extensibility of configurations so that operators can manage virtualized servers that have their own forms of configurations.

\section{Virtual-Network Configuration Acquisition Function}

\subsection{Architecture}

A virtual-network management system that manages the configurations of the whole data-center network (including virtualized servers and switches) was developed. These configurations are called the "system configuration."

Fig 3 shows the architecture of the proposed system. The proposed system consists of a server-information acquisition function, a switch-information acquisition function, and an XML merge function. These functions collect configurations from virtualized servers and switches and aggregate them into a system configuration. Operators then add link information if needed.

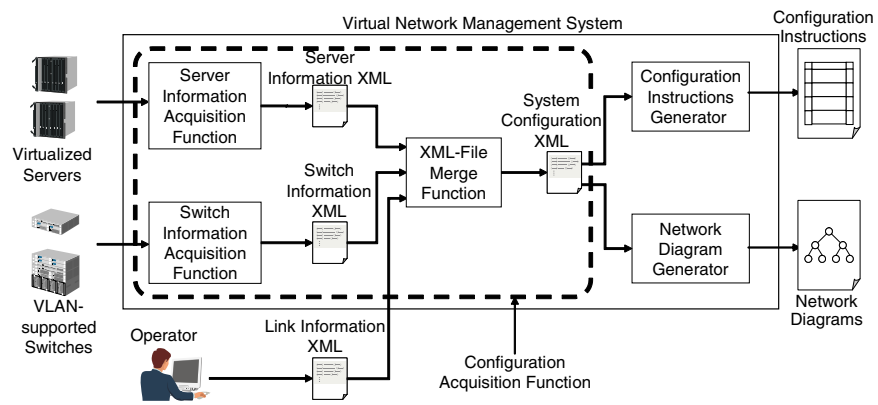

Fig. 3. Structure of the virtual network management system

The proposed system configuration has three characteristics. First, the system configuration is defined as an XML document. Since XML documents have a textual and structured data format, they are easily processed by programs. The XML-based system configuration can therefore improve reusability of configurations from the viewpoint of other management systems.

Second, the system configuration includes XML elements used to represent the overall network. These elements aggregate multiple elements, which are equivalent to managed network devices. The system configuration can therefore describe the overall structure of the managed network in an integrated manner.

Third, the system configuration includes the XML elements that represent the configuration of a managed node. These elements can keep the configurations specific to device type. The system configuration can therefore describe multiple types of configurations. 


\subsection{System Configuration XML}

Fig 4 and Fig 5 show the XML trees of the system-configuration. The XML tree shown in the Fig 4 has configurations elements to represent device configurations.

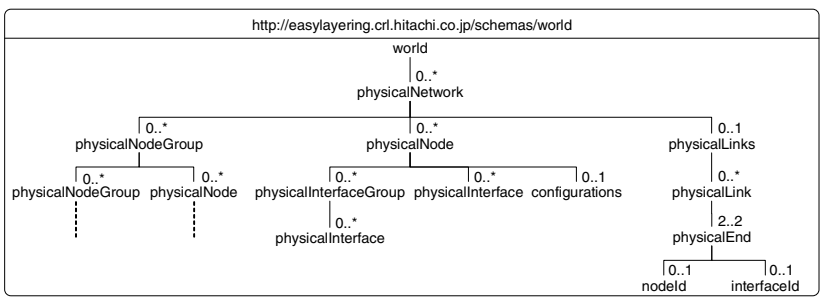

Fig. 4. XML tree of system-configuration XML
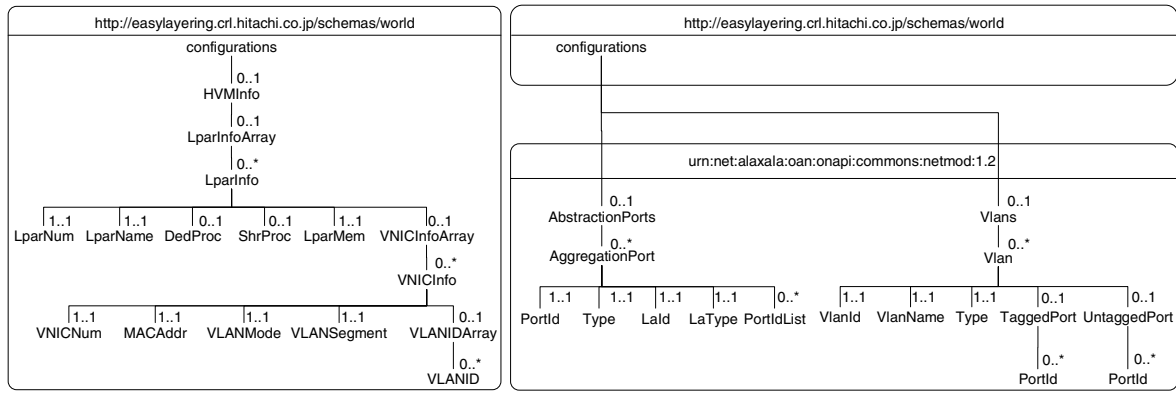

Fig. 5. XML elements for VM/VLAN configurations

The left side of Fig 5 shows the XML tree of a configurations element for a virtualized server. The LparInfo element in this XML tree has child nodes, corresponding to a name, a CPU core number, and an amount of memory of a VM. It also has a VNICInfoArray element that represents VNICs of the VM.

The right side of Fig 5 shows the XML tree of a configurations element for a switch. It has an AbstractionPorts element that represents LAG (link aggregation) 1 configurations and an Vlans element that represents VLAN configurations as the child nodes. These two elements have a set composed of AggregationPort elements and a set of Vlan elements as child nodes, respectively.

\subsection{Configuration Acquisition Function}

The server-information acquisition function, as shown on the upper side of Fig 6 , gets configurations from the virtualized servers and generates the server information XML. To generate the server information XML, this function collects the system information including the list of server blades, virtual-machine configurations, and interface/LAG/VLAN configurations through the management 


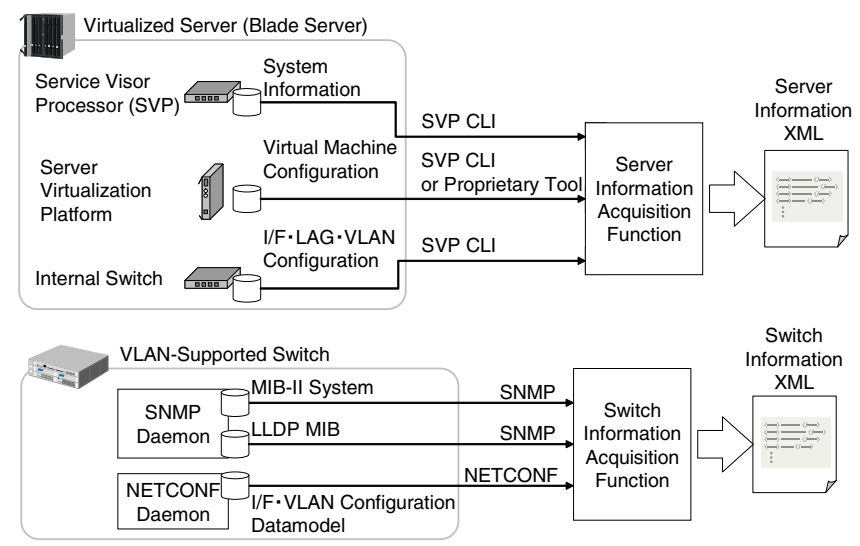

Fig. 6. Generating server/switch information XML

interface of the management module of the blade server, server-virtualization platform, and internal switch, respectively.

The switch-information acquisition function, as shown on the lower side of Fig 6. gets configurations from the daemons on the switches, which manage MIBs and NETCONF 6] configuration datamodels. This function collects the contents of the MIB-II System group 12 for the physicalNode elements. It also collects the contents of the LLDP (Link Layer Discovery Protocol) MIB [2] for the physicalLinks elements. Further, it uses the NETCONF protocol to collect NETCONF I/F and VLAN configuration datamodels for physicalinterface, AggregationPort, and Vlans elements.

The XML-file merge function gets the server-configuration XML and the switch-configuration XML, and it then aggregates them into a systemconfiguration XML. If there are multiple physicalNetwork elements with the same ID in those XMLs, this function aggregates those elements into a physicalNetwork element.

\section{Evaluation}

\subsection{Evaluation Method}

To evaluate the proposed function, we used the test network containing a servervirtualization supported blade server and four VLAN-supported switches. Five virtual machines and three VLANs were configured in this test network. In addition, a virtual-network-management-system prototype with the proposed function implemented by Perl and Java was set up. This prototype was run on a Windows Server 2003 PC, incorporating a Xeon 3GHz CPU and 2GB RAM.

We evaluated the performance of the proposed configuration-acquisition function by measuring the time the proposed function took to export a systemconfiguration XML file. Further, we evaluated the efficiency improvement for operators, by measuring the time they took to update configurations in the case 
when the operators used existing CLIs and the case when they used our prototype. The following steps are included in the measured time. First, the operator gets the VLAN configurations from devices. Second, he checks the configurations according to VLAN configuration data sheets. Finally, he updates the sheets if necessary.

\subsection{Evaluation Result}

The left side of Fig[7 shows the measurement results for the time taken to make the system-configuration XML with the prototype. The total time is $97.85 \mathrm{~s}$, which is broken down in terms of each device as blade-server chassis, $38 \%$, internal switch, $24 \%$, layer- $2 / 3$ switch, $29 \%$, and others, $9 \%$.

And, the right side of Fig 7 shows the comparative measurement result for the time taken by the operator to update the configuration sheets. The conventional method took an average $1870 \mathrm{~s}$ and the proposed method took $1126 \mathrm{~s}$; in other words, the proposed method reduced the time by $39.8 \%$ on average. Moreover, the proportions of the times for each task are not significantly different in the two cases (i.e., "by hand" and "by tool").

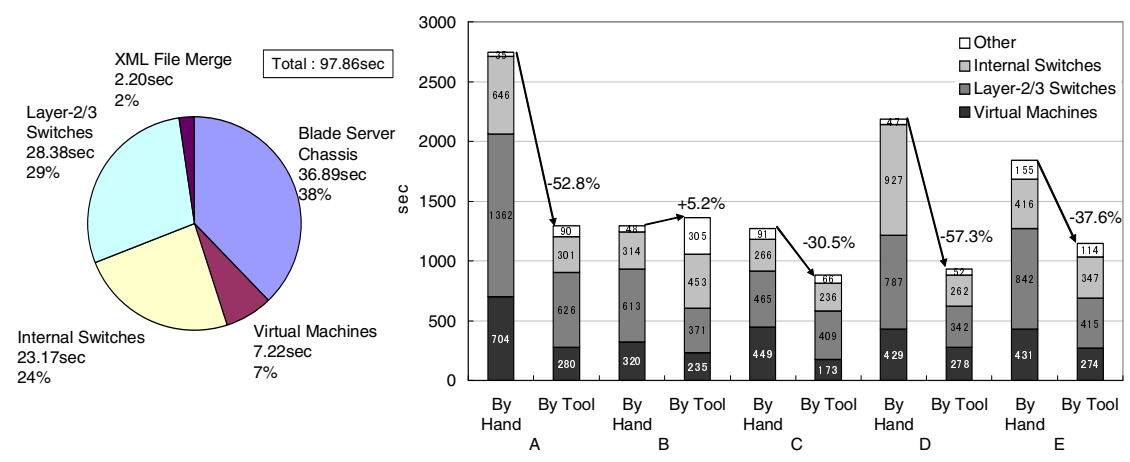

Fig. 7. Measurement result of evaluation

\subsection{Discussion}

The virtual-network-configuration acquisition function improved the efficiency of the configuration-update processes performed by operators without regard to device type. The reason for this improvement is considered to be as follows. Operators can get all information required to update the configuration sheets just by accessing the system-configuration XML.

Further, the proposed function would be effective in the case of dynamic changes of configurations, such as VM migrations. In such case, operators can easily acquire the latest configurations by using the proposed function at the notification about configuration changes from devices.

From a scalability point of view, the proposed function should be enhanced. The reason is that the number of queries to the devices increases in proportion 
to the number of managed devices in networks. On the other hand, a query to a device is independent from those to the other devices. Thus, parallelizing the queries is effective to suppress the time to collect configurations.

Further, the proposed function can be also applied to manage HPC platforms. Especially, it is useful in the case that HPC users use their own isolated platforms by means of server virtualization. However, to specify the user of a VLAN, we must develop the additional function that acquire user-information from an external authentication function and manage the user-VLAN mappings.

\section{Conclusion}

A configuration-acquisition function for virtual-network management systems was developed. This function collects configurations from virtualized servers and switches and integrates the configurations into the system-configuration XML.

The performance and effectiveness of the proposed function was evaluated by using a prototype of a virtual-network management system. In particular, it took 97.85 s to collect the configurations of five VMs and three VLANs . Furthermore, when operators used the prototype, the function reduced the time taken by operators to update the configuration documents for the test network by $39.8 \%$ on average. The results show that the proposed configuration-acquisition function is effective for improving the configuration-management process for virtual networks in data centers and HPC platforms.

\section{References}

1. Link Aggregation. IEEE Std 802.1AX-2008 (November 2008)

2. Station and Media Access Control Connectivity Discovery. IEEE Std 802.1AB-2005 (May 2005)

3. Virtual Bridged Local Area Networks. IEEE Std 802.1Q-2005 (May 2006)

4. Virtual Ethernet Switch Profile Version 1.0.0. DMTF Profile DSP1097 (October 2010)

5. Case, J., Mundy, R., Partain, D., Stewart, B.: Introduction and Applicability Statements for Internet Standard Management Framework. IETF RFC 3410 (December 2002)

6. Enns, R.: NETCONF Configuration Protocol. IETF RFC 4741 (December 2006)

7. Huang, W., Liu, J., Abali, B., Panda, D.K.: A Case for High Performance Computing with Virtual Machines. In: Proc. The 20th ACM International Conference on Supercomputing (ICS 2006), Queensland (June 2008)

8. Israel, R., Fang, Y., Cohen, P., Eichen, E.: Configuration Management of Large IP Telephony Networks. In: Proc. 2000 IEEE/IFIP Network Operations and Management Symposium, Honolulu, pp. 435-446 (April 2000)

9. Ito, M.: Japan Datacenter Services 2010-2013 Forecast and 2009 Review: Customer Perceptions and Needs. Market Analysis \# JP2542801S (February 2010)

10. Kim, M.S., Leon-Garcia, A.: Autonomic Network Resource Management using Virtual Network Concept. In: Ata, S., Hong, C.S. (eds.) APNOMS 2007. LNCS, vol. 4773, pp. 254-264. Springer, Heidelberg (2007)

11. Kusano, K.: Japan Datacenter Network Infrastructure 2009-2012 Forecast. Market Analysis \# JP209203S (July 2009)

12. McCloghrie, K., Rose, M.T.: Management Information Base for Network Management of TCP/IP-based internets: MIB-II. IETF RFC 1213 (March 1991) 